\title{
CLINICAL EVALUATION OF THE EFFICACY OF SILVER DIAMINE FLUORIDE IN ARRESTING ACTIVE DENTIN CARIES IN PRIMARY MOLARS
}

\author{
Mahy Rady ${ }^{1}$, Salah El Beheri ${ }^{2}$, Shimaa Mahfouz ${ }^{3}$, Gihan EL Dessouky ${ }^{4}$, Ghada Abd El Hameid El baz ${ }^{5}$
}

DOI: $10.21608 / d s u .2021 .77947 .1070$

Manuscript ID: DSU-2106-1070

\section{KEYWORDS}

Atraumatic restorative

technique, Early childhood

caries, Silver diamine fluoride.

- E-mail address: mahyabdelazim@hotmail.com

1. Assistant Lecturer, Pediatric Dentistry and Dental Public Health Department, Faculty of Dentistry, Misr international University.

2. Professor of Pediatric Dentistry and Dental Public Health Department, Faculty of Oral and Dental Medicine, Cairo University.

3. Associate Professor of Pediatric and Preventive Dentistry and Dental Public Health Department, Faculty of Dentistry, Suez Canal University

4. Professor of Oral Radiology, Faculty of Dentistry, Suez Canal University.

5. Professor of Pediatric and Preventive Dentistry and Dental Public Health Department, Faculty of Dentistry, Suez Canal University.

\begin{abstract}
Introduction: The atrauamatic restorative technique or approach has achieved considerable interest worldwide. Silver Diamine Fluoride (SDF) controls active carious lesions and helps in preventing further progression of the disease. Aim of the study: To evaluate and compare the efficacy of conventional glass ionomer cement (Fuji IX) and silver diamine fluoride in arresting active dentin carious lesions in primary molars through clinical evaluation. Materials and Methods: Sixty primary molars, with active dentin carious lesion (scores 4 or 5) according to ICDAS II in 25 children (4-7 years) were included in the present study. All selected teeth were treated with modified atraumatic restorative technique (ART). The treated teeth were divided into two groups according to the restorative material: Group 1 was restored with Fuji IX while Group II was restored with SDF and Fuji IX. The treated teeth were evaluated clinically for specific parameters and followed- up for 3, 6,9 and 12 months. Results: When compared to Group II regarding all the tested clinical criteria (spontaneous pain, mobility, sensitivity to percussion and sinus or swelling); there was a statistically significant decrease in success rate within both group I $(P$-value $<0.001$, effect size $=0.367)$ and group II $(P$-value $<0.001$, effect size $=0.187)$. According to Kaplan-Meier survival analysis, the mean survival time for Group I was 9.9 months and for Group II was 11.2 months. There was no statistically significant difference between the survival of the two materials within the time frame of the present study (P-value $=0.170)$. Conclusion: It could be concluded that $38 \%$ SDF offers an easy and efficient alternative treatment for early childhood caries in young children in comparison to traditional restorative procedures with better longevity, treatment outcome and prognosis.
\end{abstract}

\section{INTRODUCTION}

Dental caries is an international public health challenge especially among young children. It is not self-limiting like other infectious diseases. It begins and rapidly progresses in patients of high risk who are left untreated. Its consequences can affect the quality of life of these children. Toddlers experiencing dental caries at early stage have greater probability of subsequent caries in both the primary and permanent dentition ${ }^{(1)}$.

The atraumatic restorative treatment (ART) technique or approach has achieved considerable interest worldwide, especially for its 
applications in developing countries. It is a painfree restorative procedure that involves no local anesthesia or drilling. The ART approach involves caries removal by hand instruments, followed by restoration with highly viscous glass ionomer cement which provides chemical adhesion to the tooth surface, fluoride release and biocompatibility. The adjacent pits and fissures can also be sealed simultaneously using glass ionomer cement inserted under finger pressure ${ }^{(2)}$.

Over the past fifty years, many changes have occurred in development and availability of restorative materials for children. Fluoride releasing and chemical bonding properties of glass ionomer cements are well known. However, poor physical properties such as tendency to undergo surface crazing, low fracture resistance, and esthetics limit its use ${ }^{(3)}$.

Glass ionomer cements' desirable properties include fluoride release, antimicrobial activity, a coefficient of thermal expansion similar to that of tooth structure, and a physicochemical bond with tooth structure providing excellent sealing ability. These qualities make glass-ionomer cements an excellent restorative material for patients who are caries-active or have high caries risk factors ${ }^{(4)}$.

A generation of glass ionomer, GC Fuji IX GP, has been developed which may offer some benefits to the dental patients, especially children. It contains fluoride, adheres to tooth structure without the need of any additional bonding system, has adequate strength and can be finished and polished in one visit $^{(5)}$

Recently, there are a number of nonoperative approaches that are feasible and effective for caries management in children. These include tooth brushing with fluoride toothpaste and the use of various fluoride agents for slowing down the progression of cavitated carious lesions, such as silver diamine fluoride (SDF) which has gained much attention due to its good results in arresting dentine caries $^{(6)}$ -

Silver Diamine Fluoride is a clinically applied treatment that controls active carious lesions and helps in preventing further progression of the disease. Its application is easy, noninvasive, affordable, and safe. Although it stains the lesions dark as it arrests them, it provides clinicians with an additional tool for caries management when esthetics is not a primary concern ${ }^{(7)}$. Therefore, this study was performed to clinically evaluate and compare the efficacy of conventional glass ionomer cement (Fuji IX) and silver diamine fluoride in arresting active dentin carious lesions in primary molars.

\section{SUBJECTS AND METHODS}

\section{A. Study settings and design}

The study was a randomized clinical trial. Children who participated in the study were selected from the patients attending the outpatient clinic of Pediatric Dentistry Department, Faculty of Dentistry, Suez Canal University, Egypt. Approval of the Research Ethical Committee of the Faculty of Dentistry, Suez Canal University (47/2017) was obtained before starting the study.

\section{B. Study population}

Twenty-five children with 60 primary molars who met the study inclusion criteria were selected to participate in the study. Their age ranged from 4-7 years having at least two primary molars with active carious dentin lesion (scores 4 or 5) according to International Caries Detection and Assessment System (ICDAS II) ${ }^{(8)}$ 


\section{Eligibility criteria}

\section{Inclusion criteria:}

1. The children were apparently healthy and medically free children of both genders

2. Their age ranged from 4-7 years

3. They should have at least two bilateral primary molars with active carious dentin lesion (scores 4 or 5 )

4. Uncooperative children who are negative rating (2) according to Frankel's behavior rating scale.

5. Absence of clinical evidence of pulp involvement or degeneration ${ }^{(9)}$

- No history of spontaneous pain or pain that is not relieved by analgesics.

- No pain on percussion

- No premature hyper mobility.

- No presence of an abscess or a sinus.

6. Absence of any radiographic changes (as revealed by pre-operative periapical radiograph)_

- Internal root resorption.

- External root resorption

- Inter-radicular or periapical bone destruction (radiolucency).

\section{Exclusion criteria:}

1. Patients with allergy to silver as revealed after taking history and performing skin test.

2. Patients with ulcerative gingivitis

Personal data, medical and dental histories were recorded, clinical dental and radiographic examination was done and recorded using diagnostic sheet. In addition, preoperative digital images were saved in the active patient card on a personal computer.

Clinical examinations were performed for all patients prior to any clinical operative procedures. Eligible consented participants with carious primary molars were randomly assigned to either side (right or left). The selected teeth were equally divided into control and intervention groups (30 molars each) according to a sequence generated on a Microsoft Excel sheet, where the control group (I) and experimental group (II) were simply randomized. The power of the sample was calculated using the G-Power 3.13 power analysis software.

Control group (I) was restored with conventional glass ionomer (Fuji IX GP FAST). While the experimental Group (II) was treated with silver diamine fluoride then restored with conventional glass ionomer (Fuji IX GP FAST). The superficial soft decayed infected dentin tissues on the cavity walls were removed by sharp spoon excavator using the atraumatic restorative technique. All the prepared cavities were thoroughly cleaned with water spray and dried by air. The prepared cavities were assigned into one of the study groups according to the restorative material used.

Patient follow up was carried out after 3 months, 6 months, 9 months and 12 months. The teeth were evaluated each follow up visit for the following clinical features: spontaneous pain (presence or absence), tooth mobility (presence or absence), sensitivity to percussion (presence or absence), and examination of any changes of the mucobuccal folds. If the tooth exhibited any one of above clinical features, then the procedure of placing the restoration was considered as failure (F); otherwise, it was regarded as clinical success $(\mathrm{S})$. Statistical analysis was performed with IBM SPSS Statistics for Windows, Version 23.0. Armonk, NY: IBM Corp. 


\section{RESULTS}

The present study was conducted on 25 patients; 16 boys (64\%) and 9 girls (36\%). The mean (Standard deviation) values for age were 4.9 (0.9) years with a minimum of 4 and a maximum of 7 years old. The study utilized 60 primary molars; 14 primary first molars (23.3\%) and 46 primary second molars (76.7\%). Twelve molars (20\%) were maxillary primary molars and 48 molars (80\%) were mandibular primary molars. The results of the current study are presented as comparison between group I (Fuji IX) and group II (silver diamine fluoride and Fuji IX) regarding the following clinical criteria: spontaneous pain, tooth mobility, sensitivity on percussion and changes in the mucco-buccal fold. In addition, survival analysis of both study materials was carried out.

Concerning the changes within each group; regarding spontaneous pain throughout the follow up period, there was a statistically significant decrease in success rate within group I ( $P$-value $<0.001$, effect size $=0.367)$ and group II ( $P$-value $<0.001$, effect size $=0.187)$. There was a significant increase in number of cases showing spontaneous pain throughout the study period in both groups at successive follow up visits till 9 months. However, from 9 to 12 months, there was no change in the success rate. (Figure 1).

As concerning the changes within each group regarding tooth mobility throughout the study period, there was a statistically significant decrease in success rate within Group I ( $P$-value $<0.001$, effect size $=0.355)$ and Group II $(P$-value $<0.001$, effect size $=0.187$ ). There was a significant increase in number of molars showing mobility in group I compared to group II after 3 months, from 3 to 6 as well as 6 to 9 months. However, from 9 to 12 months, there was no change in success rate between both groups (Figure 2).

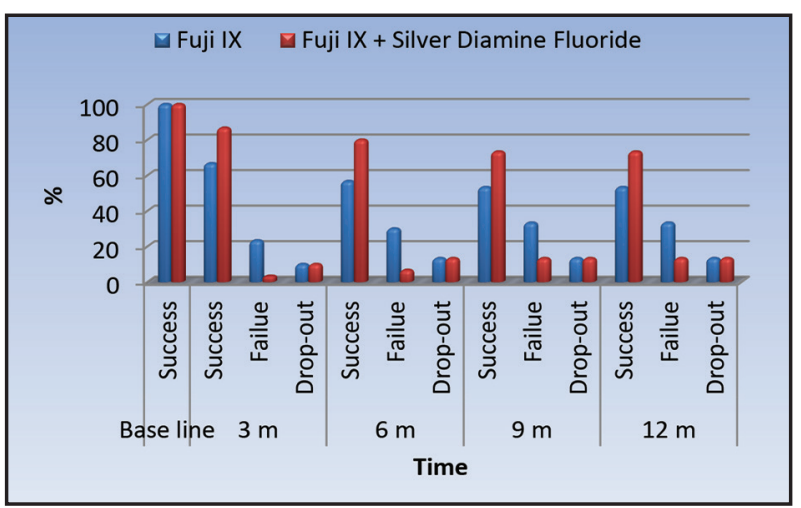

Fig. (1) Bar chart representing success and failures regarding spontaneous pain evaluation in the two groups

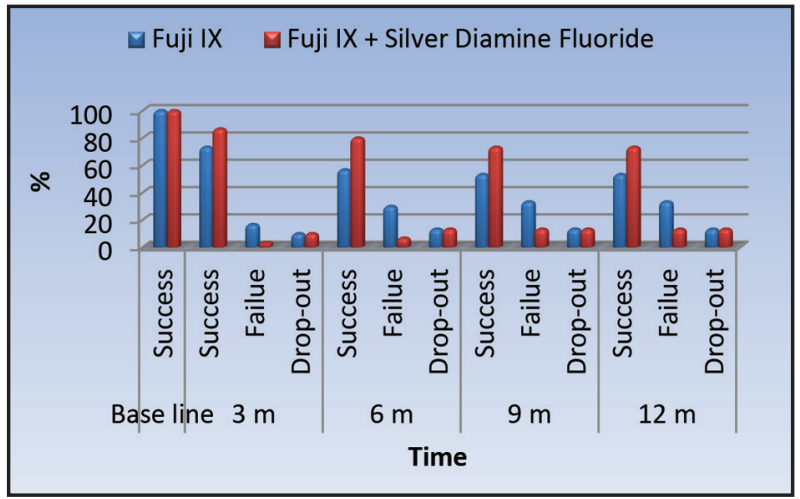

Fig. (2) Bar chart representing success and failures regarding tooth mobility evaluation in the two group

As regards the sensitivity to percussion within each group, there was a statistically significant decrease in success rate regarding sensitivity to percussion within group I ( $P$-value $<0.001$, effect size $=0.355)$ and group II $(P$-value $<0.001$, effect size $=0.187)$. In both groups, there was a decrease in success rate after 3 months, from 3 to 6 as well as from 6 to 9 months, but from 9 to 12 months, there was no change in the number of cases showing sensitivity to percussion (Figure 3).

As regards the changes within each group in the mucobuccal fold, there was a statistically significant decrease in success rate within group I ( $P$-value $<0.001$, effect size $=0.355)$ and group II $(P$-value $<0.001$, effect size $=0.187)$. In both groups, there 
was an increase in number of cases showing changes in the muccobuccal fold throughout the follow up periods except from 9 to 12 months, where there was no statistically significant change in number of cases showing changes in the muccobuccal fold (Figure 4).

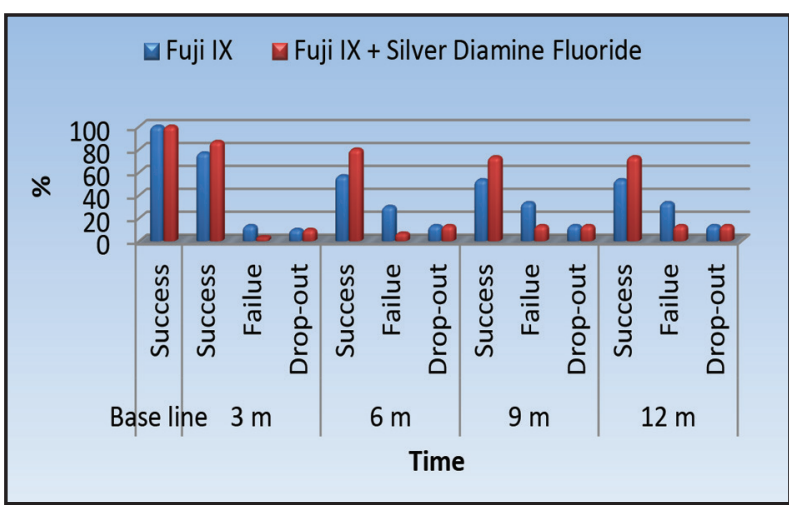

Fig. (3) Bar chart representing success and failures regarding sensitivity to percussion evaluation in the two groups

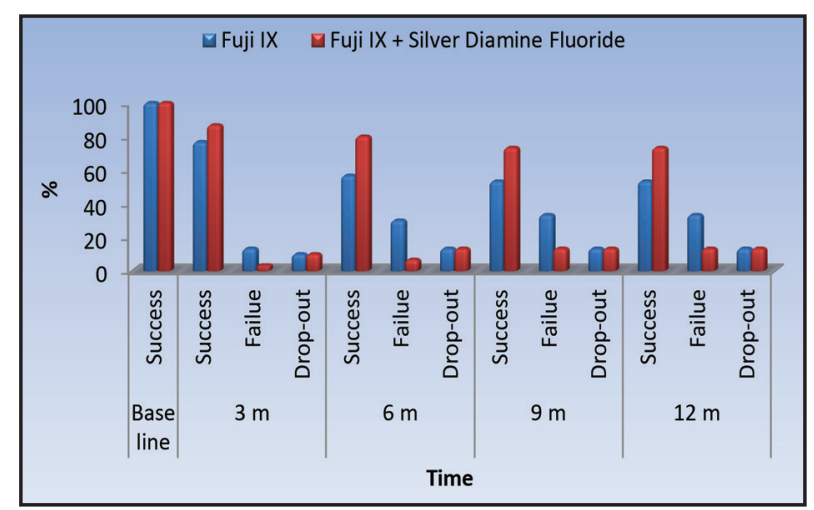

Fig. (4) Bar chart representing success and failures regarding change in muco-buccal fold evaluation in the two groups

According to Kaplan-Meier survival analysis, the mean survival time for group I was 9.9 months with $95 \%$ Confidence Interval (8.5 - 11.2) months. The mean survival time for group II was 11.2 months with $95 \%$ Confidence Interval (10.5 - 12) months. There was no statistically significant difference between the survival of the two materials within the time frame of the present study $(\mathrm{P}$-value $=0.170)$. (Table 1 and Figure 5)

Table (1) Survival rate of both study materials

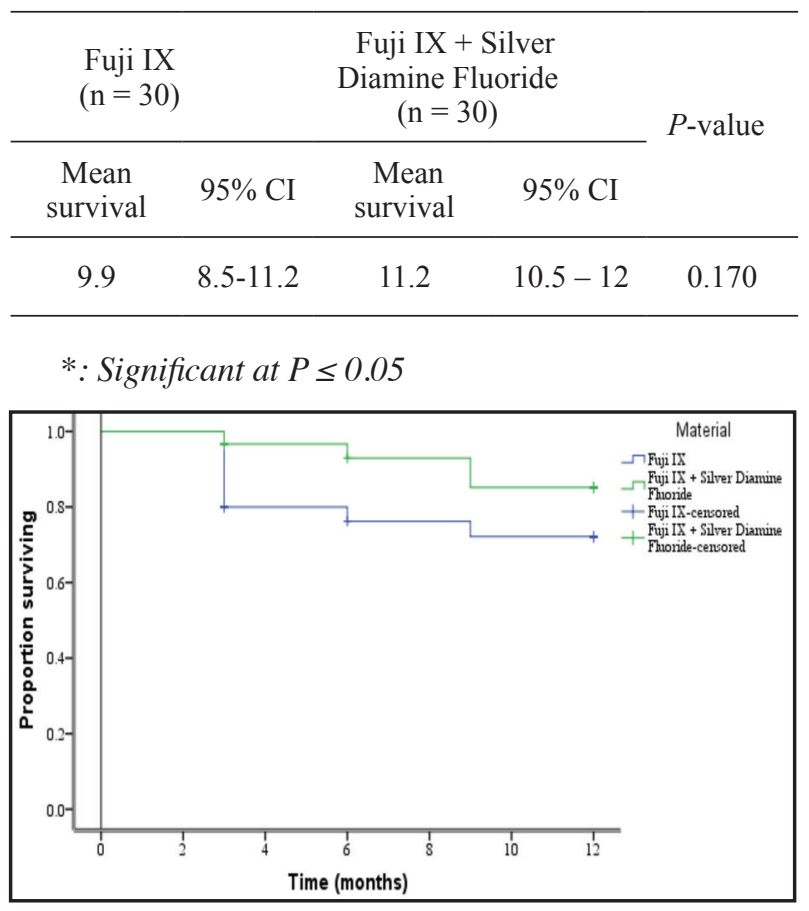

Fig. (5) Survival curve of both study materials

\section{DISCUSSION}

Early childhood caries (ECC) is one of the most prevalent diseases in children worldwide. Poor oral hygiene or insufficient dental plaque removal leads to the rapid progression of ECC. There are various methods to prevent and arrest dental caries in children, such as fluoridated agents ${ }^{(1)}$. Among these caries arresting methods, topical application of (SDF) has been receiving more and more attention due to its low cost and simplicity in treatment ${ }^{(5)}$. In the current study, we compared a modified ART technique, using Fuji IX GIC with the application of $38 \% \mathrm{SDF}$ as restorative materials since both are common restorative materials used for $\mathrm{ART}^{(9)}$. 
GIC Fuji IX was used as a final restorative material for all treated primary molars in both groups which agrees with Dos Santos and Shahid et al ${ }^{(10,11)}$ It is claimed that this cement has good chemical adhesion to the tooth structure, good biological compatibility, good aesthetics and its coefficient of linear thermal expansion is similar to that of teeth, can be finished and polished in one visit, in addition, it has also been claimed to release fluoride. SDF was found to release fluoride and help in the deposition of silver phosphate to restore the mineral content, resulting in rehardening of the tooth structure. The fluoride released from silver diamine can penetrate better into dentin than into enamel due to greater amount of protein substrate, carbonates and phosphates in dentin for reaction ${ }^{(\mathbf{1 2})}$.

In the present study, we removed the superficial soft decayed tissues found on cavity walls (infected dentin) of the selected carious primary teeth by spoon excavator leaving hard discoloration (Dentin sealing technique). Hand excavation, as a mechanical form of selective caries removal was adopted as it is capable of removing most of the infected carious dentin. Moreover, following recommendations of the International Caries Consensus Collaboration Meeting, carious tissue should be removed merely to promote conditions for durable dental restorations ${ }^{(\mathbf{1 0})}$. Moreover, Zhao et al ${ }^{(13)}$ concluded that annual application of either SDF solution or high fluoride-releasing glass ionomer can arrest active dentin caries in primary teeth. In addition, they recommended that increasing the frequency of application to every 6 months that can increase the caries arrest rate of SDF application. In agreement with our clinical findings, several in situ and in vitro studies have shown that fluoride released from GIC may slow down the progression of dental caries lesions in adjacent tooth surface, However, its clinical effectiveness for adjacent caries prevention awaits clinical trials ${ }^{(9)}$.
Regarding the clinical results, there was a statistically significant decrease in success rate within Group I when compared to Group II regarding all the tested clinical criteria (spontaneous pain, mobility, sensitivity to percussion and sinus or swelling) denoting progression of caries to reach the pulp. It is apparent that the range of caries arrest is very wide, indicating that a proportion (that varies depending on the study) of the lesions receiving treatment will not become arrested.

The results of our study are in agreement with the findings of a systematic review conducted by Niederman et al ${ }^{(14)}$ who revealed that the use of $38 \%$ SDF for caries reduction, when applied once, ranged from $96 \%$ reduction in caries for deciduous teeth to a $55.6 \%$ reduction in caries for newly erupted permanent molars.

Dos Santos et al ${ }^{(10)}$ compared the effectiveness of SDF with temporary restorations in the arrest of caries. They found that $30 \%$ SDF was 1.73 times more effective in arresting caries than an interim restorative technique after 6 and 12 months.

Moreover, multiple systematic reviews concluded that SDF application successfully arrests dental caries in children ${ }^{(15,16,17)}$ and the use of SDF has been shown to be approximately $89 \%$ (from 49 to 138\%) more effective in arresting dental caries in primary teeth than other active treatments or placebo.

Although it has also been demonstrated that for dental caries arrest, bi-annually SDF applications are superior to yearly SDF applications ${ }^{(13)}$, however, the ideal interval for SDF applications is still a matter of debate and deserves further investigation ${ }^{(18)}$.

Regarding the survival rate results of both tested materials, the mean survival time for group I was 9.9 months with 95\% Confidence Interval (8.5 - 11.2) months while the mean survival time for group II was 11.2 months with $95 \%$ Confidence 
Interval (10.5-12) month. The material used in group II improves outcome oftreatment and its prognosis is better than the material used in group I, indicating that group II SDF+ Fuji IX has better longevity and prognosis than group I (Fuji IX only) in ART technique.

\section{CONCLUSION}

Based on the results of the current study, it could be concluded that $38 \%$ SDF offers an easy, highly efficient, and well-accepted nonsurgical alternative treatment for early childhood caries in young children in comparison to traditional restorative dental treatment.

\section{REFERENCES}

1. Uribe S. Early childhood caries risk factors. EBD 2009; 10: 37-38.

2. De Amorim R, Leal S and Frencken J. Survival of atraumatic restorative treatment (ART) sealants and restorations: A meta-analysis. Clin Oral Invest 2012; 429-441.

3. Mali P. and Deshpande S. Microleakage of restorative materials an in vitro study. Indian Soc Pedodo Dent 2016; 24:15-28.

4. Mickenautsch S, Yengopal V. Caries-preventive effect of glass ionomer and resin-based fissure sealants on permanent teeth: an update of systematic review evidence. 2011; BMC Res Notes 4:22-27.

5. Singl T, Pandit IK, Srivastava N, Gugnani N \& Gupta M. An evaluation of microleakage of various glass ionomer based restorative materials in deciduous and permanent teeth: An in vitro study. Saudi Dent J 2012; 24: 35-42.

6. Edward C M L. and Duangporn D. Non-restorative Approaches for Managing Cavitated Dentin Carious Lesions. In:Soraya C L, Eliana MT, Pedc Rest Dent. Springer, Cham, 2019. p. 141-160.
7. Zafar M. \& Ahmed N. Therapeutic roles of fluoride released from restorative dental materials. Res Rev Flour 2015; 48: 184-194.

8. Rosenblatt A, Stamford T, Niederman R. Silver Diamine Fluoride: Caries “Silver Fluoride Bullet". J Res Dent 2009; 88: 116-125.

9. Hallett K, O'Rourke P. Early childhood caries and infant feeding practice. Community Dent Health 2002; 19:237-242.

10. Dos Santos V Jr, de Vasconcelos F, Ribeiro A, Rosenblatt A. Paradigm shift in the effective treatment of caries in schoolchildren at risk. Int Dent J. 2012; 62: 47-51.

11. Shahid S, Hassan U, Billington RW, Hill RG and Anderson P. Glass ionomer cements: Effect of strontium substitution on esthetics, radiopacity and fluoride release. Dent Mat J 2014; 30: 308-313.

12. Liodra J, Rodriguez A, Ferrer B, Menardia V, Ramos T. Efficacy of silver diamine fluoride for caries reduction in primary teeth and first permanent molars of schoolchildren: 36-month clinical trial. J Dent Res 2005; 84:721-724.

13. Zhao I, Gao S, Hiraishi N. Mechanisms of silver diamine fluoride on arresting caries: a literature review. Int Dent $\mathbf{J}$ 2018; 68: 67-76.

14. Niederman $\mathrm{R}$ et al. A model for extending the reach of the traditional dental practice: the Forsyth Kids program. J Am Dent Assoc 2008 139: 1040-1050.

15. Duangthip D, Jiang, M, Chu CH, Lo EC. Restorative approaches to treat dentin caries in preschool children: Systematic review. EJPD 2016, 17, 113-121.

16. Gao S, Zhao I, Hiraishi N. Clinical trials of silver diamine fluoride in arresting caries among children: A systematic review. EJPD 2016; 1: 201-210.

17. Chibinski AC, Wambier LM, Feltrin J, Loguercio AD, Wambier DS, Reis A. Silver diamine fluoride has efficacy in controlling caries progression in primary teeth: a systematic review and meta-analysis. Caries Res. 2017; 51:527-541.

18. AAPD. Policy on Early Childhood Caries (ECC): Classifications, Consequences, and Preventive Strategies. 2016. 